\title{
Size- and Temperature-control Optical Direct/indirect Band Tuning in Layered Compounds: Band Gap Engineering
}

M. Miah ( $\nabla$ m.miah@griffith.edu.au )

Griffith University Griffith Sciences

\section{Research Article}

Keywords: Layered compounds, Band gap engineering, Eg

Posted Date: June 28th, 2021

DOl: https://doi.org/10.21203/rs.3.rs-542129/v1

License: (c) (i) This work is licensed under a Creative Commons Attribution 4.0 International License.

Read Full License 


\title{
Size- and temperature-control optical direct/indirect band tuning in layered compounds: band gap engineering
}

\author{
M. Idrish Miah ${ }^{1,2}$ \\ ${ }^{1}$ Queensland Micro- and Nano-technology Centre, Griffith University, Nathan, Brisbane, \\ QLD 4111, Australia. \\ ${ }^{2}$ Department of Physics, University of Chittagong, Chittagong-4331, Bangladesh. \\ Email:m.miah@griffith.edu.au
}

\begin{abstract}
The X-ray diffraction (XRD) is studied in thermally evaporated cadmium iodide $\left(\mathrm{CdI}_{2}\right)$ thin films with various thicknesses. The grain size, calculated from the $\mathrm{XRD}$, is found to increase with increasing the thickness of the film, while the reflectivity and refractive index decease with increasing the wavelength of the exciting light. The optical absorption spectra show both allowed direct and indirect interband transitions across a fundamental gap in $\mathrm{CdI}_{2}$. It is found that both indirect and direct band gap $\left(E_{\mathrm{g}}\right)$ decrease with increasing the thickness of the film and that the indirect $E_{\mathrm{g}}$ is lower than the direct $E_{\mathrm{g}}$ by an amount of about $0.7 \mathrm{eV}$. The direct $E_{\mathrm{g}}$ is also decreased with increasing both the grain size and temperature. However, the temperature dependence of $E_{\mathrm{g}}$ follows the Varshni relation. Our results highlight the possibility of engineering or tuning the $E_{\mathrm{g}}$ of $\mathrm{CdI}_{2}$ by controlling the thickness of the film, grain size as well as temperature.
\end{abstract}

Keywords: Layered compounds; Band gap engineering; $E_{\mathrm{g}}$

\section{Introduction}

Cadmium iodide $\left(\mathrm{CdI}_{2}\right)$ is one of the group IIB iodides and typically dihalides as $\mathrm{MX}_{2}$. This is an important compound having a layered structure with an infinite hexagonal 
sheet of $\mathrm{Cd}$ atoms sandwiched between two similar sheets of I atoms. The $\mathrm{Cd}$ atoms are octahedrally coordinated to constitute the basic layer by a strong covalent bonding within $a b$-plane forming a sheet like structure and these sheets are arranged along the third direction $c$ to form a three-dimensional solid. A weak van der Waals bonding between these sheets leads to polytype structures having a number of polytypes, as many as 200 [1].

$\mathrm{CdI}_{2}$ is a wide-band gap semiconductor having optical band gap $\left(E_{\mathrm{g}}\right)$ near a weak insulator and with highly anisotropic chemical bonds. [2]. Band structure calculations for $\mathrm{CdI}_{2}$ have shown [3-5] large anisotropy of the space charge density distribution causing high anisotropy of optical spectra and the presence of a direct $E_{\mathrm{g}}$ and a slightly smaller indirect $E_{\mathrm{g}}$. $\mathrm{CdI}_{2}$ is central symmetric or centrosymmetric in the bulk. However, the anisotropic behaviour of $\mathrm{CdI}_{2}$ makes it polar, so a strong electron-phonon interaction is expected. This favours the local noncentrosymmetricity for the observation of the second harmonic generation (SHG) in $\mathrm{CdI}_{2}$ nanostructures [6-8]. The role of photo-induced electron-phonon anharmonic interactions in SHG was explored [9].

The thermoluminescence and photoluminescence studies revealed that this material processes higher order optical nonlinearity [10-14]. Materials exhibiting higher order optical nonlinearities have received much attention due to their potential in applications such as optical switching, amplification and limiting [15]. The ferroelectric was studied by Kityk et al. [16]. This is most probably the first extensive study on $\mathrm{CdI}_{2}$ thin films.

These interesting structural and nonlinear optical properties of $\mathrm{CdI}_{2}$ make it a better candidate for the intensive studies [17]. The various aspects of the layered compounds have thus been investigated for the better understanding and advancing new device ideas. Band gap engineering is a powerful technique for the design of new semiconductor materials and devices $[18,19]$. In this process, the transport properties of electrons and holes could be independently and continuously tuned for a given application or device (e.g. photomultiplier). The experimental demonstration on the band gap engineering of layered hexagonal $\mathrm{SnSe}_{2}$ nanostructured thin films by varying the thickness was first 
given by Mukhokosi et al. [20]. They showed that for a 50-nm thick film, the band gap $E_{\mathrm{g}}$ is about $2.04 \mathrm{eV}$, whereas $E_{\mathrm{g}}$ is approximately $1.2 \mathrm{eV}$ for the bulk or $1200-\mathrm{nm}$ thick film. Their study highlights the possibility of engineering the band gap of layered $\mathrm{CdI}_{2}$ by controlling the thickness of the films. In a recent study, $\mathrm{CdI}_{2}$ thin films were grown by chemical bath deposition and their crystalline structure and linear optical properties including optical band gap were controlled by changing the $\mathrm{pH}$ of the chemical bath at room temperature [21]. Dependence of $E_{\mathrm{g}}$ on residual stress in $\mathrm{ZnI}_{2}, \mathrm{CdI}_{2}$ and $\mathrm{HgI}_{2}$ films was also studied [22]. However, studies focusing on the optical band gap and its related properties are quite limited for $\mathrm{CdI}_{2}$ thin films. As the new generation of devices with unique capabilities is emerging from this approach, it would be worthy to carry out a systematic and intensive study on this issue in $\mathrm{CdI}_{2}$ thin films. Here, in the present investigation, we study the dependence of the direct and indirect $E_{\mathrm{g}}$ on grain size, film thickness and temperature. In addition, we also study the reflectivity and refractive index of the films as function of wavelength of the exciting light.

\section{Experimental}

One of the convenient methods to grow group IIB iodides is thermal evaporation. Thin films $\mathrm{CdI}_{2}$ were grown on glass substrates at room temperature by evaporation using a molybdenum boat. The typical glass substrates were $2.5 \mathrm{~cm} \times 5 \mathrm{~cm}$ sizes. The starting material was high purity (99.99\%) analar grade powder that was palletized for evaporation. All the $\mathrm{CdI}_{2}$ films used in this study were grown at the vacuum of $10^{-6}$ Torr. The film thickness was monitored by a quartz crystal thickness monitor during evaporation and subsequently confirmed by DEKTAK IIA surface profiler measurements. The structural study was carried out by X-ray-diffraction (XRD) analysis (PHILIPS, Model PW-1830). The film composition of the as grown films was found to be stoichiometric. However, no significant deviation from stoichiometric film composition was observed for heat-treated films or films grown at substrate temperatures up to a couple hundred ${ }^{0} \mathrm{C}$. The optical absorption measurements were carried out in the UV-Visible region using a Shimadzu UV-260 spectrophotometer. For the low temperature measures the samples were mounted in a liquid nitrogen temperatureregulated cryostat. The temperature of the sample may be varied between 80 and $300 \mathrm{~K}$. 


\section{Results and discussion}

We studied XRD and determined grain size $(D)$ and film thickness $(L)$. We carried out measurements on a large number of samples. As there existed a number of XRD and TEM/SEM based structural, compositional and morphological studies on this material in the literature $[19,20]$, we are not interested to report them here. We only focus on the XRD data which are relevant to the objectives of the present study. The grain size $D$ of the material was calculated using the well-known Debye-Scherrer formula [23]

$D=\frac{0.9 \lambda}{H \cos \theta}$

where $H$ is the half width of XRD lines, $\lambda$ is its wavelength and $\theta$ is the half diffraction angle (i.e. Bragg angle) of $2 \theta$. Film thickness was obtained from the relation $L=D^{3} N$, where $N$ is the number of crystallites per unit area. Grain size $D$ as a function of film thickness $L$ is shown in Fig. 2. As can be seen, $D$ increases with increasing film thickness. As can be seen the grain size varies from $110 \mathrm{~nm}$ to $160 \mathrm{~nm}$ for the film thickness $50 \mathrm{~nm}$ to $550 \mathrm{~nm}$. The dislocation density $(S)$ can also be obtained from the relation $S=1 / D^{2}$.

We performed optical absorption and reflectance measurements on a large number of samples with different thicknesses. Figure 3 insert shows the reflectance $(r)$ as a function of $\lambda$ for a film of thickness $L=360 \mathrm{~nm}$. As can be seen, $r$ decreases with increasing $\lambda$ and a maximum value of about $20 \%$ is obtained for the higher frequency. The refractive index ( $\mu$ ) was calculated using the relation [24]

$\mu=\frac{1+r}{1-r}+\sqrt{\frac{4 r}{(1-r)^{2}}-\left(\frac{\alpha \lambda}{4 \pi}\right)^{2}}$

where $\alpha$ is the absorption coefficient and is related to the extinction coefficient $(\beta)$ as $\beta=\alpha \lambda /(4 \pi)$. The refractive index as a function of wavelength is shown in Fig. 1, where 
$\mu$ decreases with $\lambda$. The refractive index varies from 2.1 to 2.6 for wavelength $\lambda=700 \mathrm{~nm}$ to $250 \mathrm{~nm}$.

For the direct type of transition, the conduction band (CB) minimum and the valance band (VB) maximum is in line on the energy $(E)$ scale in the $E$ - $k$ diagram, where $k=2 \pi / \lambda$ is the wave vector (Fig, 3). However, it deviates for the phonon-assisted indirect transition, as there are phonons involved in the process. Therefore, the band gap $E_{\mathrm{g}}$ is the energy difference between the $\mathrm{CB}$ minimum and VB maximum, or the energy difference between the $\mathrm{CB}$ and VB at $k=0$.

The absorption coefficient $\alpha$ was calculated from $\alpha=2.303 A / L$, where $A$ is the absorbance, neglecting the reflection which is insignificant near the absorption edge. The optical absorption shows both allowed direct and indirect interband transitions across a fundamental gap confirming the results of the earlier band structure calculations for the optical direct and indirect band gap $E_{\mathrm{g}}$ in $\mathrm{CdI}_{2}$ thin films. Figure 4 shows a typical absorption spectrum of the material, where the dependence of $\alpha$ on $E_{\lambda}$ is plotted near the absorption edge. Here $E_{\lambda}=h v=h c / \lambda$ is the of excited photon energy. In crystalline materials and for parabolic bands, as shown in Fig. 3, the optical transitions (either direct or indirect) can be evaluated by the relation between $\alpha$ and $E_{\mathrm{g}}$. For the direct transition

$\alpha E_{\lambda}=C_{1}\left(E_{\lambda}-E_{g}\right)^{p}$

and the indirect transition $[25,26]$

$\alpha E_{\lambda}=\frac{C_{2}\left(E_{\lambda}-E_{g}+E_{p h}\right)^{p}}{\exp \left(\Theta_{D} / T\right)-1}+\frac{C_{3}\left(E_{\lambda}-E_{g}-E_{p h}\right)^{p}}{1-\exp \left(-\Theta_{D} / T\right)}$

where $C_{1}, C_{1}$ and $C_{1}$ are fitting constants, $\Theta_{\mathrm{D}}$ is the Debye temperature, $E_{\mathrm{ph}}$ is the phonon energy assisting the indirect transition, and for a direct (indirect) type of transition $p=1 / 2$ 
or $3 / 2$ ( $p=2$ or 3 ) depending on whether the transition is allowed or forbidden. The optical band gap $E_{\mathrm{g}}$ of $\mathrm{CdI}_{2}$ thin films was estimated from Tauc plot. The Tauc technique of determining $E_{\mathrm{g}}$ is to plot a $\left(\alpha E_{\lambda}\right)^{1 / p}$ versus $E_{\lambda}$ graph and look for that value of $p$ which gives the best linear fit in the absorption. For an allowed transition $p=1 / 2(p=2)$ for a direct (indirect) type. As can be seen from Eqs. (3) and (4), there will be one (two) linear region(s) for the direct (indirect) transition. We plotted $\left(\alpha E_{\lambda}\right)^{1 / p}$ versus $E_{\lambda}$ for the films of various thicknesses and discovered both type of allowed transitions. Figures 5 and 6 show the results. A linear fit to the data of the higher wavelength region of the graph gives a direct $E_{\mathrm{g}}$ as an intercept of the vertical axis (Fig. 5). Similarly, two lines fitted to the data of the low and high wavelength regions of the graph gives the indirect type $E_{\mathrm{g}}$ (Fig. 6). Figure 7 shows the dependence of $E_{\mathrm{g}}$ on $L$ for the direct and indirect transitions. As can be seen, the optical band gap $E_{\mathrm{g}}$ for both direct and indirect types varies with the thickness of the film. This variation looks like quadratic in nature. The direct (indirect) $E_{\mathrm{g}}$ is found to vary from about 3.05 to $3.65 \mathrm{eV}(2.50$ to $3.20 \mathrm{eV})$ for the variation of film thickness from $400 \mathrm{~nm}$ to $5.5 \mu \mathrm{m}$. This variation consists well with that for the other layered hexagonal $\mathrm{SnSe}_{2}$ nanostructured thin films [20]. The increase of $E_{\mathrm{g}}$ with $L$ may be attributed to the formation of crystal defects, which can produce localized states that change the effective Fermi level due to an increase in carrier concentrations [14,26]. Tyagi et al. [22] studied the variation of $E_{\mathrm{g}}$ of $\mathrm{CdI}_{2}$ thin films, grown on glass substrate, with residual stress parameter and showed that there exists a threshold tensile stress above which $E_{\mathrm{g}}$ decreases linearly. They found a direct $E_{\mathrm{g}}$ of $3.60 \mathrm{eV}$ for the film. The possible optical transition in these films was found to be allowed direct transition with the $E_{\mathrm{g}}$ decreasing from 3.76 to $3.57 \mathrm{eV}$ with increasing the film thickness [27]. Our values agree well with these. A slight decease of our values might be responsible for the growth conditions which affect the formation of the defects. This could reduce the concentration of localized states in band structure. However, the band gap $E_{\mathrm{g}}$ for the indirect transition is lower than that of the direct transition by an amount of $\sim 0.7 \mathrm{eV}$, which is due to the phonon assisted transition. The dependence of $E_{\mathrm{g}}$ on $D$ for the direct transition is shown in Fig. 8. For the higher $D$, the dependence is seen to be linearly decreasing with increasing $D$. The observed variation, almost similar to the thickness dependence, might be due to the defect densities either line defects (e.g. dislocation) or planar defects [28]. 
Figure 9 shows the dependence of $E_{\mathrm{g}}$ on the temperature $T$ for the direct transition. The dependence is modeled by the Varshani relation [29]

$E_{g}(T)=E_{g}(0)-\frac{a T^{2}}{b+T}$

where $E_{\mathrm{g}}(0)$ is the band gap at $T=0 \mathrm{~K}$, and $a$ and $b$ are temperature independent constants. In Fig. 9, we also plot Eq. (3) using the parameters' values for the material. The plot shows the experimental data agrees well with the Varshani relation and $E_{\mathrm{g}}$ decreases with increasing $T$. This is because of band-shifting effect at higher temperature, supporting earlier investigations [30]. This study shows a way of tuning the band gap in layered structured cadmium iodide compounds by controlling the temperature and film thickness/grain size.

\section{Conclusions}

$\mathrm{CdI}_{2}$ thin films with various thicknesses were grown by thermal evaporation and studied by XRD. Optical band gap $E_{\mathrm{g}}$ and its related properties were determined. The grain size was found to increase with increasing the thickness of the film, while the reflectivity and refractive index deceased with increasing the wavelength. The optical absorption spectra showed both allowed direct and indirect interband transitions across a fundamental gap. It was found that both indirect and direct $E_{\mathrm{g}}$ decrease with increasing thickness of the film and that the indirect $E_{\mathrm{g}}$ was lower than the direct $E_{\mathrm{g}}$, confirming the results of the earlier band structure calculations. The direct $E_{\mathrm{g}}$ was also decreased with increasing both grain size and temperature. The temperature dependence of $E_{\mathrm{g}}$, however, followed the Varshni relation. The findings resulting from this investigation suggest the possibility of tuning and engineering the $E_{\mathrm{g}}$ of $\mathrm{CdI}_{2}$ by controlling the thickness of the film, grain size as well as temperature. The results are discussed in comparison with those in the literature.

\section{Conflicts of interest}

The author declares no conflicts of interest. 


\section{References}

[1] F. Hulliger, In: Levy F, Reidel D, eds. Structural chemistry of layer-type phases. Holland: Dordrecht-Holland; 1976. p. 31-4.

[2] N. F. Mott, E. A. Davis, Electronic Processes in Non-crystalline Materials (Clarendon Press, Oxford, 1979).

[3] J. Bordas, J. Robertson, A. Jakobsson, J. Phys. C 11 (1978) 2607.

[4] J. Robertson, J. Phys. C 12 (1979) 4753.

[5] M. R. Tubbs, J. Phys. Chem. Solids 26 (1968) 1191.

[6] M. I. Miah, J. Appl. Phys. 104, 064313 (2008).

[7] M. I. Miah, J. Phys. Chem. B 113, 1652 (2009).

[8] H. Ollafsson, and F. Stenberg, Opt. Mater. 341 (2004).

[9] M. I. Miah and J. Kasperczyk, Appl. Phys. Lett. 94, 053117 (2009).

[10] Z. Spurny, Nucl. Instr. Methods 175, 71 (1980).

[11] I. M. Catalano, A. Cingolani, R. Ferrara, and M. Lepore, Helv. Phys. Acta 58 (1985) 329.

[12] F. Adduci, A. Cingolani, R. Ferrara, M. Lugara, and A. Minafra, Nuova Cimento B 38, 610 (1977).

[13] M. I. Miah, Opt. Mater. 18, 231 (2001).

[14] M. I. Miah, Phys. Lett. A 374, 75 (2009).

[15] M. I. Miah, Optik- Intern. J. Light Electron Opt. 123, 1580 (2012).

[16] I. V. Kityk, S. A. Pyroha, T. Mydlarz, J. Kasperczyk, and M. Czerwinski, Ferroelectrics 205, 107 (1998).

[17] M. I. Miah, Eur. Phys. J. B 92, 224 (2019).

[18] F. Capasso, Science 235, 172 (1987).

[19] A. Sitt, I. Hadar, U. Banin, Nanotoday 8, 494 (2013).

[20] E. P. Mukhokosi, S. B. Krupanidhi, and K. K. Nanda, Sci. Rep. 7, 15215 (2017).

[21] I. A. Kariper, J. Mater. Res. Technol. 5, 77 (2016).

[22] P. Tyagi , R. K. Mishra, N. C. Mehra, and A. G. Vedeshwar, Integ. Ferroelectr. 122, 52 (2010).

[23] R. S. Rawat, P. Arun, A. G. Vedeshwar, P. Lee, S. Lee, J. Appl. Phys. 95, 7725 
(2004).

[24] A. Sarkar, S. Chaudhuri, A. K. Pal, Phys. Stat. Sol. A 119, K21 (1990).

[25] J. J. Pankove, Optical processes in semiconductors and conductors, Prentice Hall, Englewood Cliffs, New York, 1971.

[26] J. C. Slater, Phys Rev 103, 1631 (1956).

[27] I. S. Yahia, M. Shapaan, Y. A. M. Ismail, A. M. Aboraia, E. R. Shaaban, J. Alloys Comp. 636 (2015) 317-322.

[28] M. Salimullah, P. K. Shukla, S. K. Ghosh, H. Nitta, and Y. Hayashi, J. Phys. D: Appl. Phys. 36, 958 (2003).

[29] Y. Varshni, Physica 34 (1967) 149.

[30] M. I. Miah, Spectrosc. Lett. 42,431 (2009). 


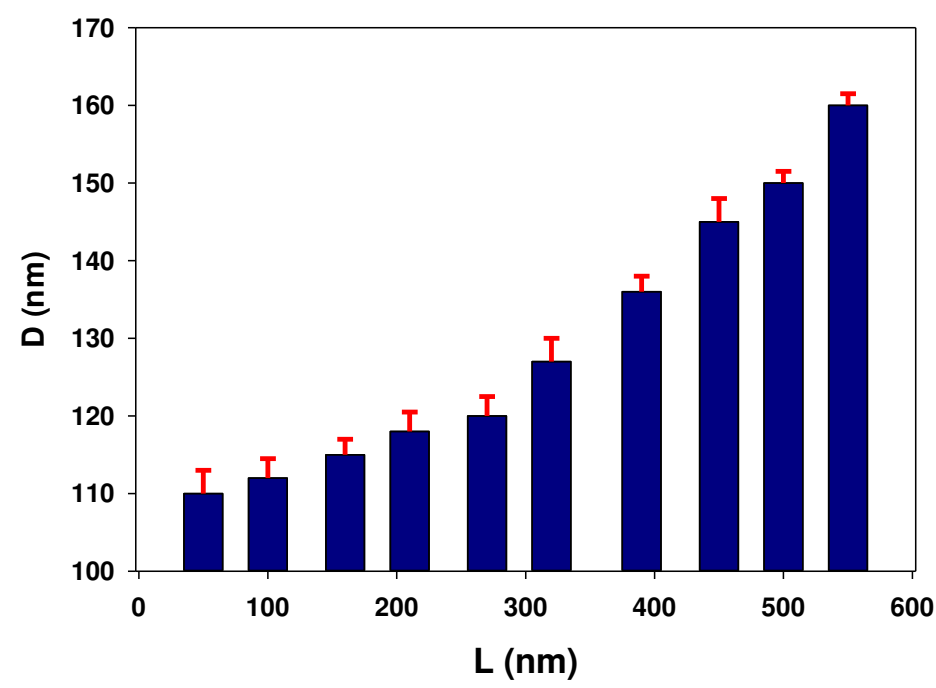

Figure 1. Grain size as a function of film thickness measured from XRD. Calculation details are given in the text. 


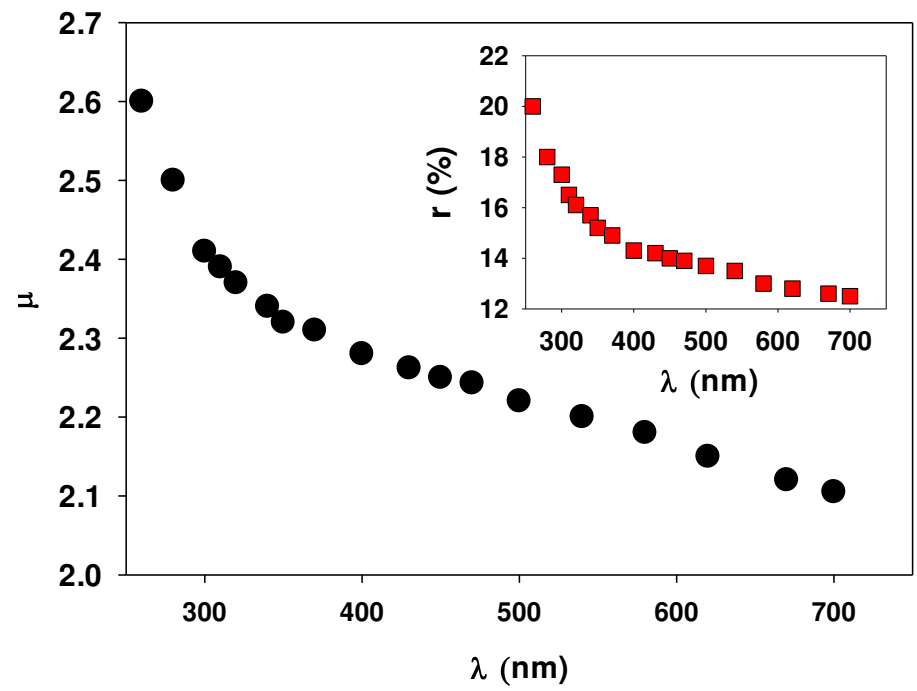

Figure 2. Refractive index as function of wavelength of the exciting light $\lambda$ for a film of thickness $L=360 \mathrm{~nm}$. Insert show the dependence of reflectivity on $\lambda$. 


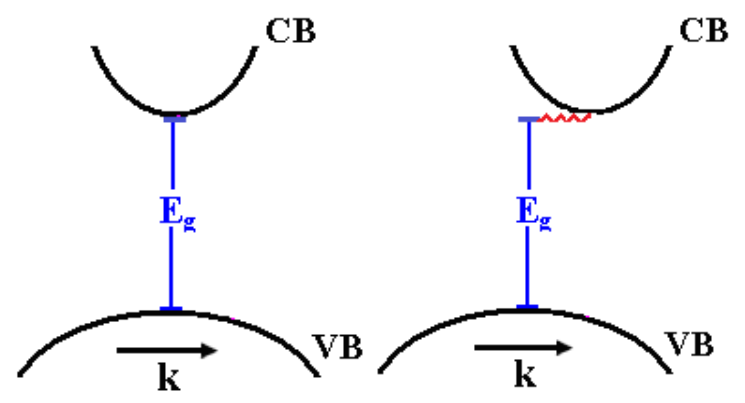

Figure 3. Direct (left) and phonon-assisted or indirect (right) band gap $E_{\mathrm{g}}$ as a function of the wave vector $k . E_{\mathrm{g}}$ is the energy difference between the conduction band (CB) minimum and the valance band (VB) maximum, or the energy difference between the $\mathrm{CB}$ and $\mathrm{VB}$ at $k=0$. In indirect $E_{\mathrm{g}}$, the phonon involvement is indicated by a wave. 


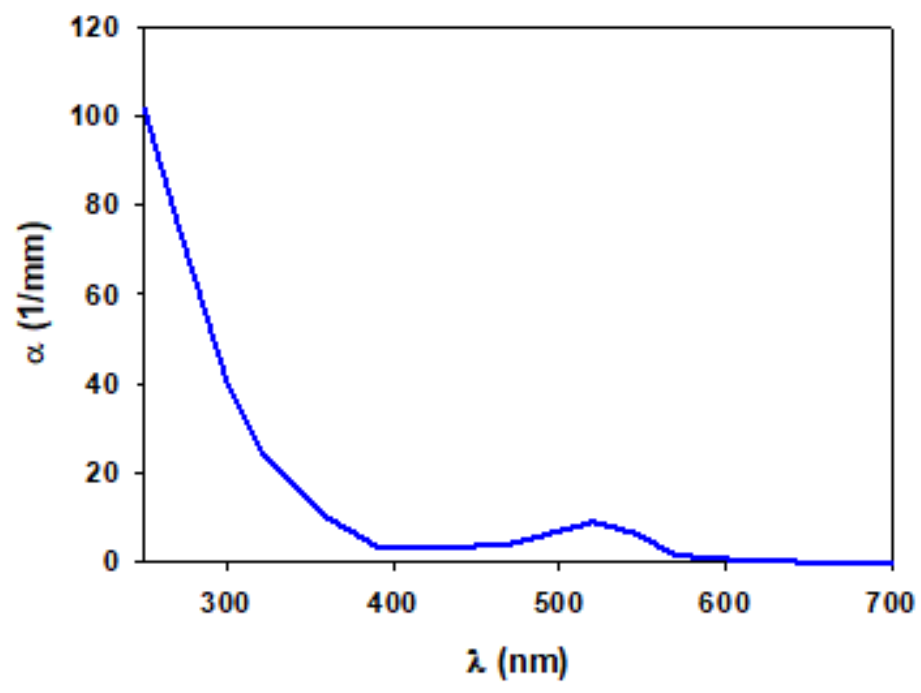

Figure 4: A typical absorption spectra. The absorption coefficient $\alpha$ was calculated from the absorbance and the film thickness. 


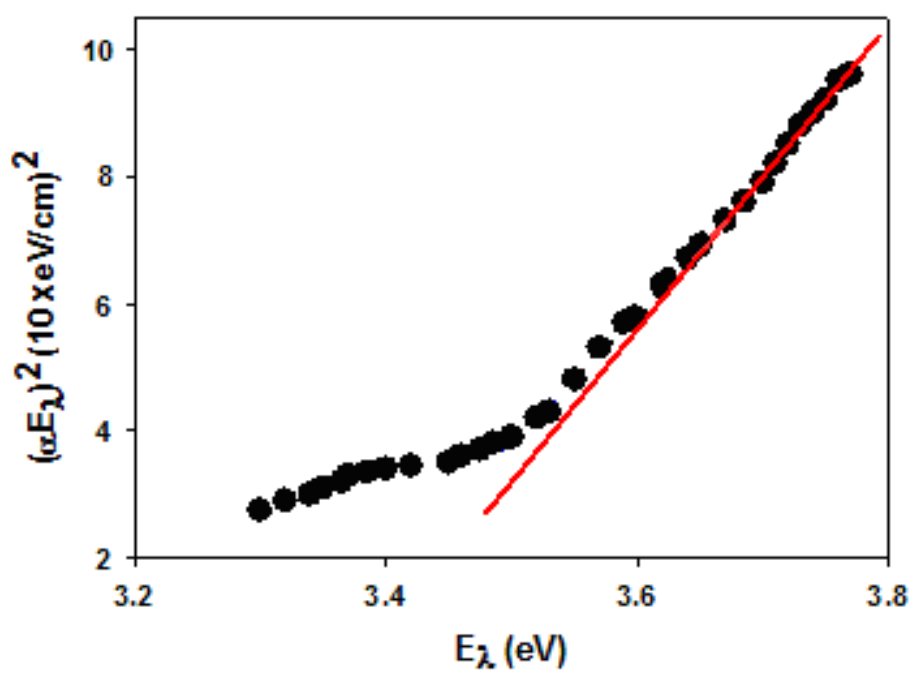

Figure 5: A plot of $\left(\alpha E_{\lambda}\right)^{2}$ versus $E_{\lambda}$ with $E_{\lambda}=h v=h c / \lambda$. Line is a linear fit to the data of the higher wavelength region of the absorbed photon energy curve. 


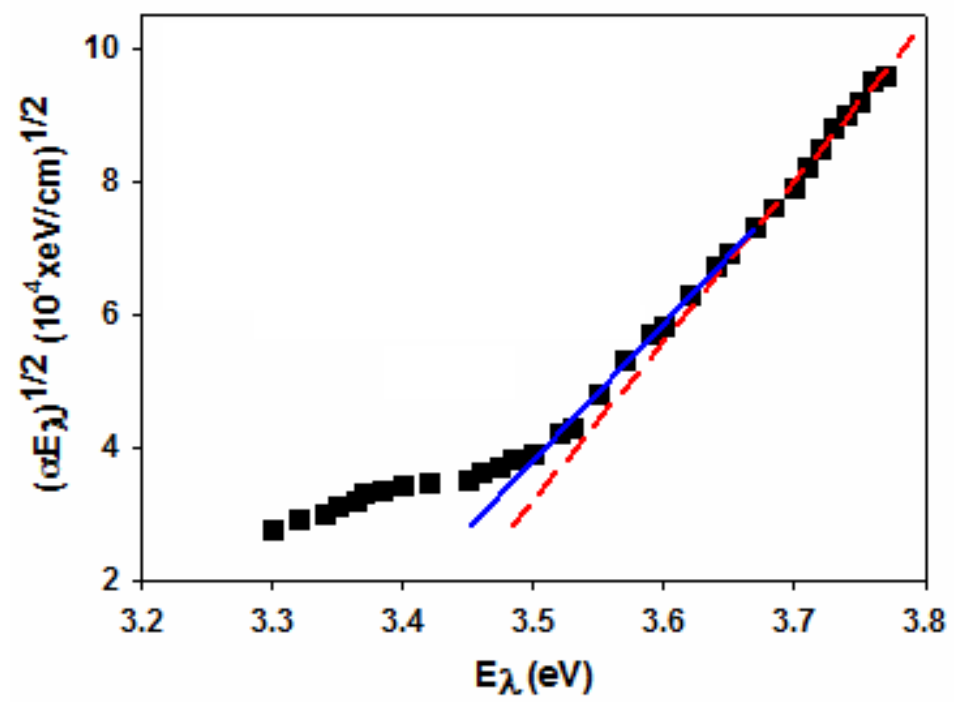

Figure 6: A plot of $\left(\alpha E_{\lambda}\right)^{1 / 2}$ versus $E_{\lambda}$. Lines are linear fits to the data of the low (solid) and high (dashed) wavelength regions of the curve. 


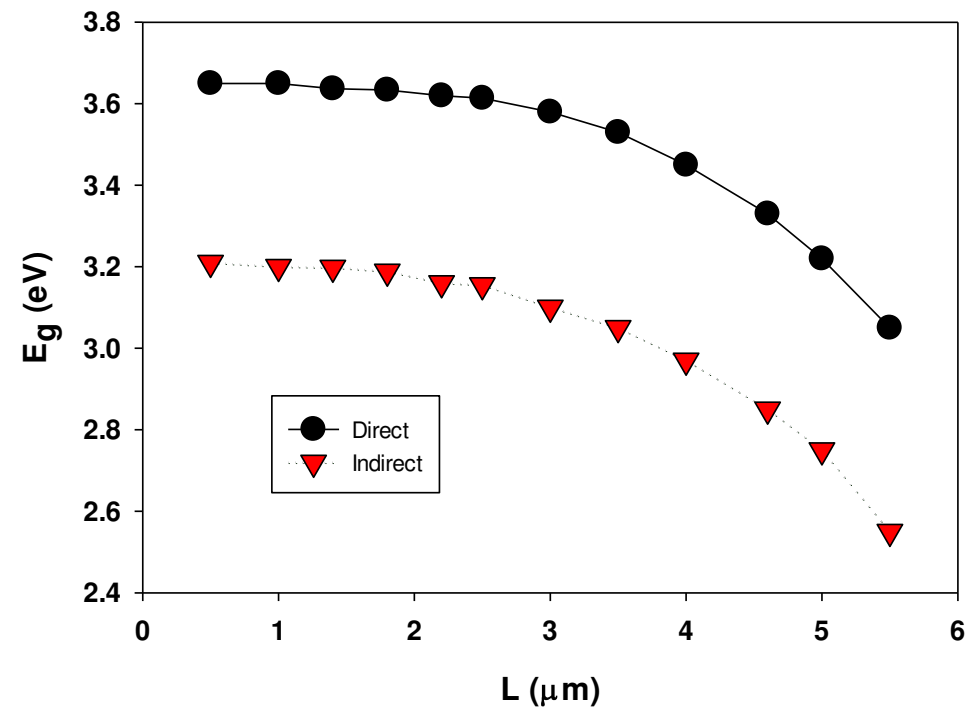

Figure 7: Dependence of $E_{\mathrm{g}}$ on $L$ for the direct and indirect transitions between parabolic bands. 


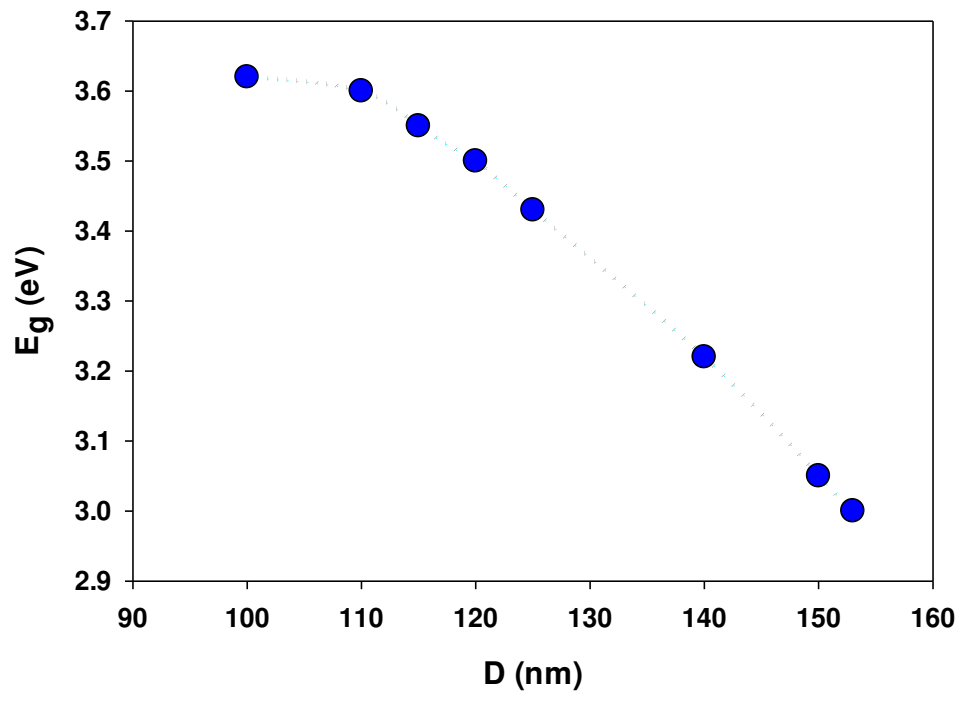

Figure 8: Dependence of $E_{\mathrm{g}}$ on $D$ for the direct transition. For the higher $D$, the dependence is linear. 


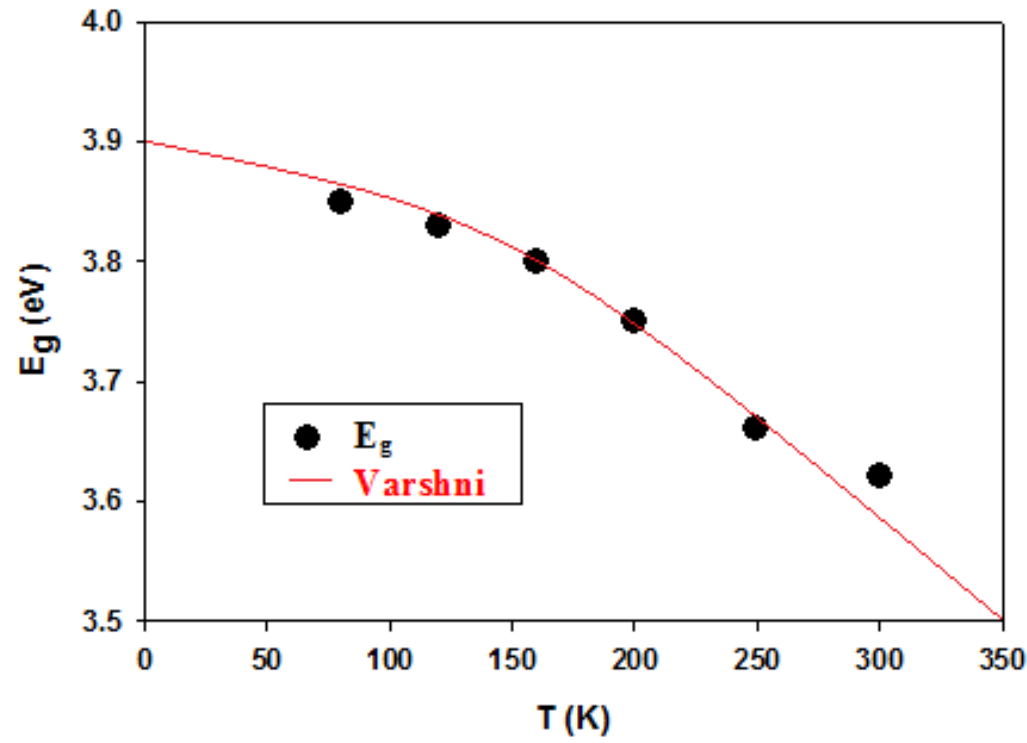

Figure 9: Dependence of $E_{\mathrm{g}}$ on $T$ for the direct transition. The solid line is a fit to the data by the Varshni relation. 